OPEN ACCESS

Edited by:

Hannelie Korf

KU Leuven, Belgium

Reviewed by:

Thierry Gustot,

Free University of Brussels, Belgium

Gianluca Matteoli,

KU Leuven, Belgium

${ }^{*}$ Correspondence:

Antonio Riva

a.riva@researchinliver.org.uk orcid.org/0000-0002-4974-2780

Gautam Mehta

g.mehta@researchinliver.org.uk

tThese authors have contributed equally to this work

Specialty section:

This article was submitted to

Molecular Innate Immunity,

a section of the journal

Frontiers in Immunology

Received: 15 October 2018

Accepted: 18 January 2019

Published: 05 February 2019

Citation:

Riva A and Mehta G (2019) Regulation of Monocyte-Macrophage Responses in Cirrhosis - Role of Innate Immune

Programming and Checkpoint

Receptors. Front. Immunol. 10:167.

doi: 10.3389/fimmu.2019.00167

\section{Regulation of Monocyte-Macrophage Responses in Cirrhosis-Role of Innate Immune Programming and Checkpoint Receptors}

\author{
Antonio Riva ${ }^{1,2 * t}$ and Gautam Mehta ${ }^{1,2,3 * t}$ \\ ${ }^{1}$ Institute of Hepatology London, Foundation for Liver Research, London, United Kingdom, ${ }^{2}$ Faculty of Life Sciences \& \\ Medicine, King's College London, London, United Kingdom, ${ }^{3}$ UCL Institute for Liver and Digestive Health, University College \\ London, London, United Kingdom
}

Many aspects of the innate immune system have been studied in cirrhosis, and abnormalities have been described supporting both a pro-inflammatory and anti-inflammatory phenotype of myeloid cells. However, the findings of these studies vary by stage of disease and methodology. The recent description of the syndrome of acute-on-chronic liver failure (ACLF) has refined our understanding of the natural history of cirrhosis. In this context, we review the regulatory mechanisms at play that contribute to the immune abnormalities described in advanced liver disease. Specifically, we review the evidence for epigenetic mechanisms regulating monocyte phenotype, and the role of checkpoint receptors on regulating innate and adaptive immune cell function.

Keywords: cirrhosis, ACLF, ARLD, ALD, alcoholic hepatitis, innate immune cell, myeloid, immune checkpoint

\section{BACKGROUND}

This is an exciting time for the field of immunotherapeutics. Advances in basic science and drug development have progressed our understanding of regulatory mechanisms of both innate and adaptive immune responses, which has directly led to novel immunotherapeutic agents. Moreover, technological advances have allowed unbiased data collection from monocyte-macrophage lineage cells, allowing a deeper understanding of their diversity and plasticity (1). The purpose of this review is to integrate these data and place them within the context of the disease landscape of cirrhosis.

Inflammation, and consequently innate immunity, plays a key role in the development of liver disease at almost every stage. For example, in the early stages, monocyte-macrophage lineage cells play a role in both the development $(2,3)$ and the resolution (4) of hepatic fibrosis. Understanding and harnessing the mechanism of fibrosis resolution by hepatic macrophages is an area of active translational research, although is beyond the scope of this review. This area has been reviewed recently by Ramachandran et al. (5).

Acute-on-chronic liver failure (ACLF) is a recently defined syndrome, describing an acute clinical deterioration on the background of cirrhosis, characterized by a rapid progression to multiorgan failure and high mortality. The CANONIC study, the largest prospective study of the natural history of cirrhosis, demonstrated that in the majority of cases (60\%) a specific trigger for the acute deterioration, such as bacterial infection or acute alcoholic hepatitis, could be identified (6). It was also apparent from this study that dysregulated inflammation is a key feature of the syndrome. The degree of systemic inflammation, determined by leukocyte count and C-reactive protein, was 
an independent predictor of the development and prognosis of ACLF. However, alongside these pro-inflammatory responses, immunodeficiency and susceptibility to infection are also features of cirrhosis and $\operatorname{ACLF}(7,8)$. The overarching term for these immune alterations in cirrhosis is cirrhosis-associated immune dysfunction (CAID), although the mechanisms that regulate these diverse and dichotomous immune responses in cirrhosis remain incompletely understood (Figure 1). Nevertheless, recent insights into immune pathobiology in cirrhosis, along with general advances in our understanding of regulation of immunity, provide opportunities for novel therapies in cirrhosis. These opportunities will be discussed in more detail in this review.

The lines of evidence describing both features of exaggerated systemic inflammation, as well as immunodeficiency in cirrhosis and ACLF have been recently reviewed elsewhere (7). From a mechanistic perspective, gut bacterial dysbiosis and the translocation of bacterial products to mesenteric lymph nodes and the systemic circulation have been suggested to play a role in the development of these immunological abnormalities in cirrhosis (7) (Figure 1). However, a clear association of gut dysbiosis and a specific immune phenotype has not yet been demonstrated. Moreover, most evidence from cirrhotic patients is from single time points, and from varying severities of liver disease, as prospectively collected data delineating the time-course of immune phenotype in cirrhosis and ACLF is currently lacking. As can be seen in Table 1, both pro- and antiinflammatory responses from monocyte-macrophage cells have been described in cirrhosis. Further discussion of the evidence behind these observations is provided below.

\section{CIRCULATING MONOCYTES}

Circulating monocytes play an important role in host defense, through initiation and regulation of inflammatory responses (18). In both humans and mice their phenotype can be divided into two main subsets: classical (pro-inflammatory) and non-classical (anti-inflammatory, pro-repair), which are distinguished by surface markers $(19,20)$. These subsets are primarily separated by their expression of CD14 (the coreceptor for bacterial lipopolysaccharide, LPS) and CD16 (a low affinity type III Fc receptor for IgG). Most circulating monocytes, around 90\%, are classical $\mathrm{CD}^{-} 6^{-}$monocytes expressing high levels of CD14 $\left(\mathrm{CD}_{1} 6^{-} \mathrm{CD} 14^{+}\right)$. The remainder $\mathrm{CD}_{16}{ }^{+}$monocytes are further separated based on the expression of $\mathrm{CD} 14$ among $\mathrm{CD}_{1} 6^{+} \mathrm{CD} 14^{+}$intermediate monocytes and $\mathrm{CD} 16^{+} \mathrm{CD} 14^{\text {lo }}$ non-classical monocytes. Similar subsets are found in mice using the Ly6C, CCR2, and CX3CR1 markers, with

Abbreviations: ACLF, Acute-on-chronic liver failure; AH, Alcoholic hepatitis; ARLD, Alcohol-related liver disease; CD, Cluster of differentiation; CTLA-4; Cytotoxic T-lymphocyte associated protein 4; DAMP, Damage/Danger-associated molecular pattern; DC, Decompensated cirrhosis; IFN, Interferon; IL, Interleukin; MAIT, Mucosal-associated invariant T cells; NK, Natural killer cells; NKT, Natural killer T cells; PAMP, Pathogen-associated molecular pattern; PD-1, Programmed death 1; PD-L1, Programmed death ligand 1; SC, Stable cirrhosis; TIM-3, T-cell immunoglobulin and mucin domain 3 (also known as Hepatitis A virus cellular receptor 2, HAVCR2); TNF: Tumor necrosis factor classical Ly6C ${ }^{\text {hi }} \mathrm{CCR} 2^{+} \mathrm{CX} 3 \mathrm{CR} 1^{\text {int }}$ monocytes and non-classical

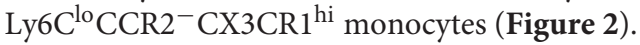

Conceptually, our understanding of the natural history of cirrhosis has progressed over recent years with description of the syndrome of ACLF, which describes patients with cirrhosis who progress from stable or decompensated cirrhosis to a rapid decline in liver function and extra-hepatic organ failure following a superimposed "hit". As can be seen from Table 1, there are few studies that examine immune cell phenotype in this stage of the disease. Monocyte dysfunction has been previously described in ACLF in cross-sectional studies, indicating skewed proportions between monocyte subsets with an increasing prevalence of anti-inflammatory monocytes able to suppress proinflammatory innate immune responses correlated with disease severity. Specifically, increased numbers of monocytes expressing the receptor tyrosine kinase Mer (MerTK) have been found in ACLF, associated with reduced pro-inflammatory responses ex vivo (17), and similarly, prostaglandin E2 (PGE2) levels have been found to be elevated in ACLF and implicated in the antiinflammatory monocyte phenotype (16) (Figure 2). However, an overarching mechanism for the change in monocyte phenotype in ACLF is currently lacking.

\section{CIRCULATING MONOCYTES RESPOND TO SUPERIMPOSED LIVER INJURY BY ALTERING THEIR PHENOTYPE AND FUNCTION}

The superimposed "hit" in ACLF, on the background of cirrhosis, has been suggested to represent an acute liver insult such as gut bacterial translocation, sepsis, alcoholic hepatitis or druginduced liver injury (DILI), leading to hepatocyte cell death and the release of damage/danger-associated molecular patterns (DAMPs) (8). Therefore, a possible hypothesis for the change in circulating monocyte phenotype in ACLF is that it represents a regulatory response to this superimposed liver injury (Figure 2).

The traditional dogma from mouse experiments has been that monocytes sequentially alter their phenotype from classical to non-classical over time, possibly in response to micro-environmental cues. For example, following injury, predominantly $\mathrm{Ly}_{6 \mathrm{C}} \mathrm{hi}$ monocytes are recruited from the bone marrow and spleen to sites of injury in a CCR2- and CCL2-dependent manner (21-23). Recent elegant experiments using deuterium labeling in humans, and adoptive transfer experiments in humanized mice, has demonstrated similar transitioning in human monocytes, particularly in response to challenge with bacterial endotoxin (24). The time course for this transition from classical to non-classical phenotype was between 1 and 5 days, with non-classical monocytes persisting for around 12 days, thus demonstrating the importance of time course in determining immune phenotype following infection or injury (Figure 2).

The relevance of these observations to liver injury has also been explored in rodent models. In a model of acetaminophen (APAP)-induced liver injury, fate-mapping studies using adoptive transfer of Ly6C ${ }^{\text {hi }} \mathrm{CX} 3 \mathrm{CR} 1^{+}$monocytes demonstrated 


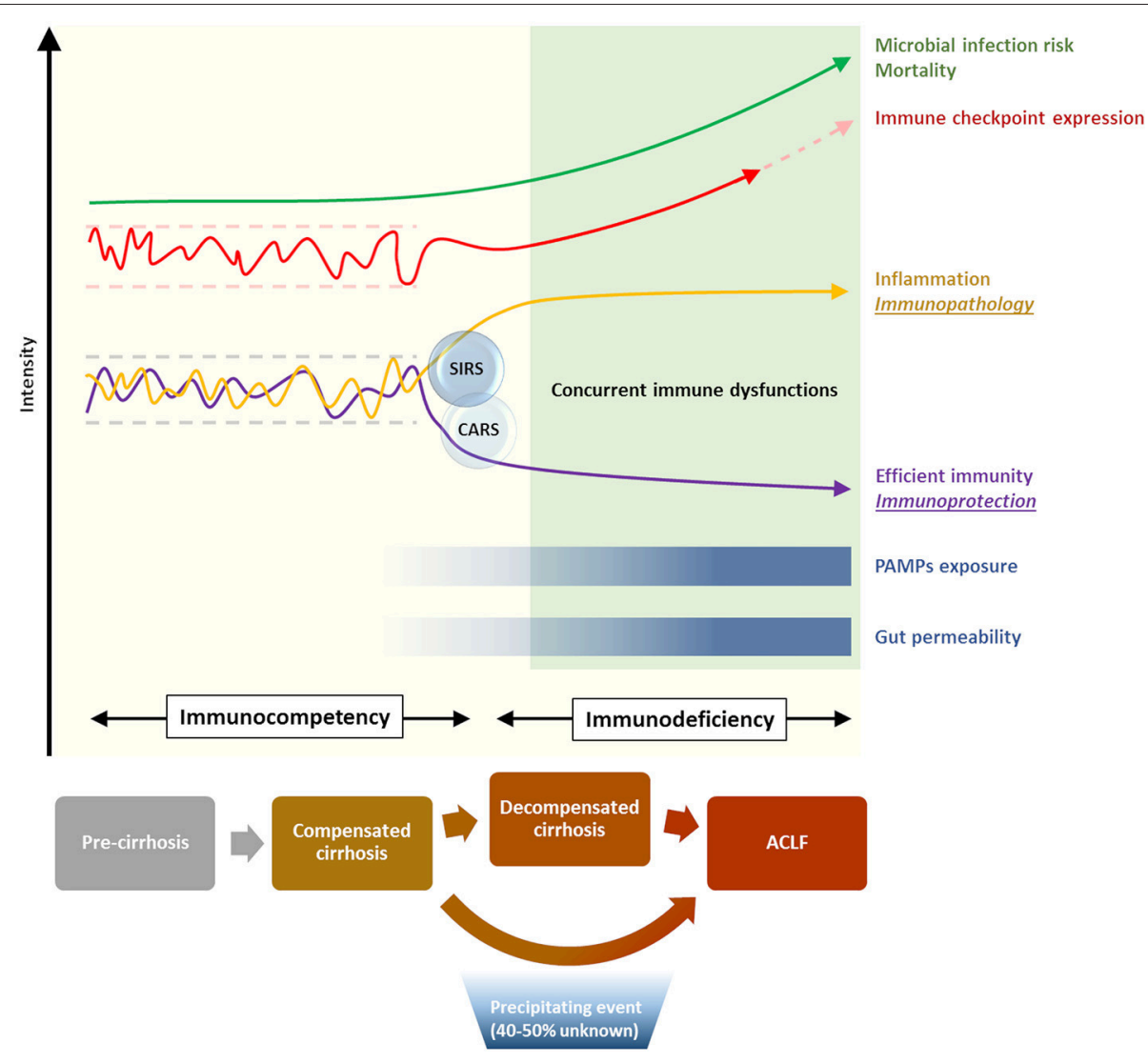

FIGURE 1 | Immunological phenomena associated with progression of cirrhosis. Increasing disease severity is accompanied by the establishment of a skewed immune profile, characterized by concurrent systemic inflammation and deficient immune protection. This state of cirrhosis-associated immune dysfunction (CAID) is crucial in increasing the risk of life-threatening microbial infection, and is in part mediated by a dysregulation of the immune checkpoint network [reviewed in (9)].

that these Ly6 $\mathrm{C}^{\text {hi }}$ monocytes differentiated into a Ly6C $\mathrm{C}^{\text {lo }}$ subset by $72 \mathrm{~h}$ following liver injury, and were cleared by $96 \mathrm{~h}$ (25). A further study by Dal-Secco and colleagues used CCR2-RFP and CX3CR1-GFP double-reporter mice with a model of sterile liver injury (26). These elegant experiments demonstrated that CCR $2^{\text {hi }} \mathrm{CX}_{3 \mathrm{CR}} 1^{\text {lo }}$ (Ly6 $\mathrm{C}^{\text {hi }}$ ) monocytes were initially recruited to the site of liver injury, and over a period of $24 \mathrm{~h}$ transitioned into a CCR $2{ }^{\text {lo }} \mathrm{CX} 3 \mathrm{CR} 1^{\text {hi }}\left(\mathrm{Ly} 6 \mathrm{C}^{\text {lo }}\right.$ ) subset that was prevalent for up to $72 \mathrm{~h}$. Therefore, it is clear that liver injury can influence the phenotype of infiltrating cells, but it remains unclear whether this occurs in cirrhosis, and to what extent this influences the phenotype of circulating immune cells. Nevertheless, most studies of monocytes in ACLF have demonstrated that monocyte dysfunction can be induced in healthy monocytes by incubation with ACLF plasma $(16,17)$ (Figure 2). Therefore, liver injury may lead to the subsequent reprogramming of circulating cells as well as infiltrating monocytes to an anti-inflammatory phenotype-this hypothesis merits further attention.

\section{DE NOVO RECRUITMENT OF ANTI-INFLAMMATORY CELLS}

A further possible mechanism is the de novo recruitment of antiinflammatory cells. In humans, a recent study in ACLF described an increased population of circulating $\mathrm{CD} 14^{+} \mathrm{CD} 15^{-} \mathrm{HLA}^{-\mathrm{DR}}{ }^{-}$ myeloid-derived suppressor cells, which lead to impaired innate and adaptive immune responses and thus contribute to the antiinflammatory phenotype of ACLF (27).

Another tier of complexity is the possible infiltration of peritoneal-derived macrophages following liver injury. Recent elegant work from Paul Kubes' lab has demonstrated that these peritoneal cells, described as $\mathrm{F} 4 / 80^{+} \mathrm{CD} 11 \mathrm{~b}^{\text {hi }} \mathrm{CD} 102^{+}$and GATA6 $^{+}$, were found to relocate to the liver within $1 \mathrm{~h}$ following liver injury, to express markers associated with tissue repair, and to be critical for survival of mice following CCl4-induced acute liver injury (28). Furthermore, using CCR2-RFP and CX3CR1GFP double-reporter mice, it was clear that these peritoneal macrophages are distinct from infiltrating peripheral blood monocytes. Further work is required to see if these cells are present in humans, and to what extent (if any) they can modify systemic immune phenotype.

\section{MONOCYTE REPROGRAMMING AND EPIGENETICS}

In a broader sense, the molecular mechanisms of monocyte reprogramming are beginning to be understood and exploited. The concept of "innate immune memory" has arisen over recent 
TABLE 1 | Monocyte-macrophages display pro- and anti-inflammatory phenotypes in end-stage liver disease.

\begin{tabular}{|c|c|c|c|}
\hline \multirow{2}{*}{\multicolumn{2}{|c|}{$\begin{array}{c}\text { Anti-inflammatory phenotype } \\
\text { Monocyte-Macrophage: }\end{array}$}} & \multirow{2}{*}{\multicolumn{2}{|c|}{$\begin{array}{c}\text { Pro-inflammatory phenotype } \\
\text { Monocyte-Macrophage: }\end{array}$}} \\
\hline & & & \\
\hline Rimola et al. (10) & $\begin{array}{l}\text { In vivo: Decreased reticuloendothelial system } \\
\text { phagocytosis in cirrhosis (DC) compared to } \mathrm{HC} \text {, by } \\
\text { tracer elimination method. This was associated with } \\
\text { increased incidence of bacterial infection. }\end{array}$ & Albillos et al. (11) & $\begin{array}{l}\text { Ex vivo: Increased activation markers (HLA-DR and } \\
\text { CD80) and intracytoplasmic TNF } \alpha \text { expression in } \\
\text { monocytes from cirrhosis (DC) compared to HC. }\end{array}$ \\
\hline Gomez et al. (12) & $\begin{array}{l}\text { In vivo: Decreased macrophage-mediated clearance of } \\
\text { lgG-coated erythrocytes in cirrhosis (mixed SC and DC). } \\
\text { This was associated with increased incidence of } \\
\text { bacterial infection. }\end{array}$ & Tazi et al. (13) & $\begin{array}{l}\text { Ex vivo: Greater increase in LPS-induced monocyte } \\
\text { TLR4 expression and TNF } \alpha \text { release from cirrhotic } \\
\text { patients compared to HC. }\end{array}$ \\
\hline $\begin{array}{l}\text { Wasmuth et al. } \\
\text { (14) }\end{array}$ & $\begin{array}{l}\text { Ex vivo: Decreased monocyte LPS-induced TNF } \alpha \\
\text { production and HLA-DR expression in ACLF compared } \\
\text { to SC. }\end{array}$ & $\begin{array}{l}\text { Gandoura et al. } \\
\text { (15) }\end{array}$ & $\begin{array}{l}\text { Ex vivo: Microarray gene expression profiling of PBMCs } \\
\text { from ARLD cirrhosis (DC) showed decreased induction } \\
\text { of type- } 1 \text { and type- } 2 \text { IFN-stimulated genes, compared to } \\
\mathrm{HC} \text { (see left column). }\end{array}$ \\
\hline
\end{tabular}

Gandoura et al. $\quad$ Ex vivo: Microarray gene expression profiling of PBMCs

(15) from ARLD cirrhosis (DC) compared to HC, showed increased induction of pro-inflammatory cytokine genes (IL-6, IL-8, TNF $\alpha$ ), but decreased induction of type-1 and type-2 IFN-stimulated genes, compared to $\mathrm{HC}$ (see right column).

O'Brien et al. (16) Ex vivo: Plasma from DC and ACLF led to decreased LPS-stimulated TNF $\alpha$ release and bacterial killing when incubated with healthy monocyte-macrophages, compared to plasma from stable cirrhosis.

Bernsmeier et al. (17)
Ex vivo: Decreased monocyte LPS-induced TNF $\alpha$ and $\mathrm{IL}-6$ production in DC and ACLF compared to stable cirrhosis. No change in ROS production. years, challenging the dogma that only adaptive immune cells have the capacity for "memory" (29). This concept describes the phenomenon whereby an innate immune cell can mount a qualitatively different response, either exaggerated ("trained immunity") or impaired (tolerance), in response to repeated challenge. As such, it is becoming clear that innate immune cells, particularly monocytes, can be reprogrammed at metabolic, epigenetic, and transcriptional levels (30). In situations with acute excessive inflammation, tolerance acts as a mechanism to dampen the inflammatory response of the host and maintain homeostasis to prevent tissue damage and organ failure $(31,32)$. Nevertheless, in conditions such as sepsis, chronic inhibitory effects in immune function can also lead to a state of deep and long-lasting immunosuppression associated with a higher risk of secondary infections and a poorer outcome (33).

Epigenetic mechanisms have been implicated in monocytemacrophage reprograming (30) (Figure 2). In response to LPS or upon pathogen exposure, monocytes and macrophages modify their histone acetylation and methylation marks, affecting gene expression patterns upon subsequent stimulation (34). For example, after LPS exposure, the repressive histone modification "H3K9 dimethylation" (H3K9me2) is noted at the promoter regions of $\operatorname{IL}-1 \beta$ and $\operatorname{TNF} \alpha(35,36)$. Potential molecular mechanisms include increased expression of histone demethylases and deacetylases following LPS exposure $(37,38)$, and several of these mechanisms have also been involved in the reprogramming of intracellular metabolic activities affecting the balance between glycolysis and fatty acid oxidation $(39,40)$ (Figure 2). These pathways are potentially targetable: inhibitors of the histone deacetylases sirtuin 1 and 2 (SIRT1/2) have shown efficacy in reversing immune paralysis in mouse models of sepsis $(41,42)$. Similarly, long non-coding RNAs have been shown to be mediators of a "switch" in monocyte phenotype in sepsis and are also potentially targetable through antisense nucleotide strategies (43). These mechanisms deserve attention in cirrhosis and ACLF.

\section{IMMUNE CHECKPOINTS}

A further level of regulation is through interaction with adaptive immune cells and regulation of signaling through immune checkpoint receptors. Immune checkpoints constitute a complex array of regulatory receptors and ligands that are expressed on the surface of both innate and adaptive immune cells. Both costimulatory and a relatively larger set of inhibitory checkpoint pathways have been described, and it is the fine balance between all these positive and negative signals that is responsible for the physiological regulation of the fate and direction of ongoing immune responses. The expression of these regulatory 


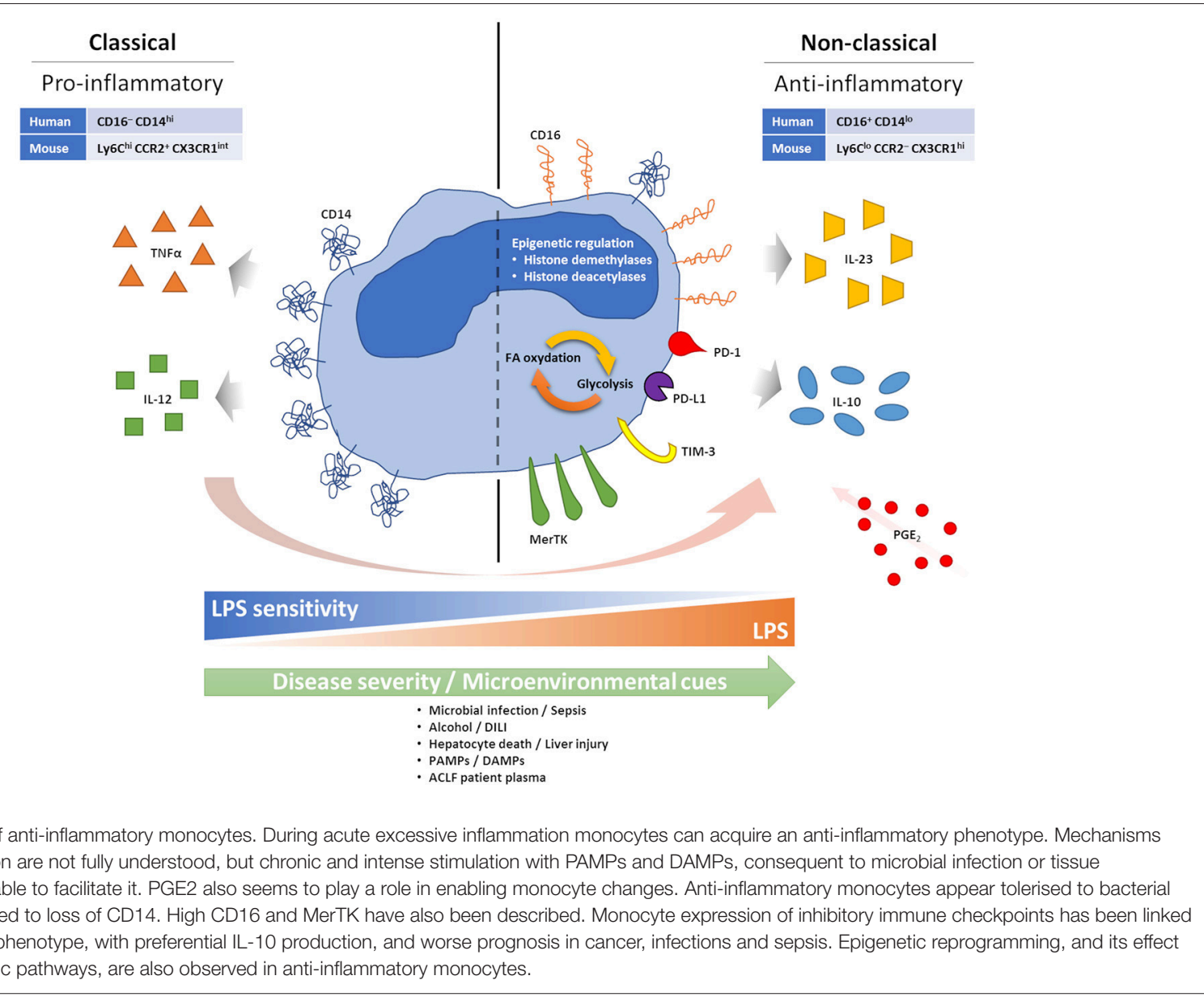

pathways is both anatomically and temporally coordinated in order to facilitate the initiation and the termination of immune responses. However, in situations where the inflammation or the antigenic stimulation persist (such as sepsis, endotoxemia, or chronic infections) inhibitory checkpoints remain upregulated and this overwhelming negative signaling leads to immune cell exhaustion and immunosuppression. Amongst the most characterized inhibitory checkpoints, PD-1 and CTLA4 (with their respective ligands $\mathrm{PD}-\mathrm{L} 1$ and $\mathrm{CD} 80$ ) have demonstrated to be novel, effective and safe immunotherapeutic targets for cancer, and new monoclonal blocking antibodies for TIM-3 are also currently in clinical development or tested in clinical trials [reviewed in (9)].

Most checkpoint pathways have been first characterized as regulators of T-cell immunity, but it is now clear that their effects are not limited to T cells only. For instance, PD-1 is known to also cause B and NK cell functional suppression [reviewed in (9)], and a study in HIV patients demonstrated that monocytes can express PD-1 upon bacterial exposure. These PD- $1^{+}$monocytes secrete suppressive IL-10 upon PD-1 engagement (Figure 2), and either PD-1 or IL-10 receptor blockade in these patients can reverse adaptive HIV-specific T-cell exhaustion(44) (Figure 2). Expression of PD-1 and PD-L1 on monocytes has also been associated with increased mortality in septic patients $(45,46)$, while expression of TIM-3 on monocytes has been linked to a more aggressive tumor phenotype in gastric cancer patients (47), a reduced pathogen clearance in malaria (48), preferential production of IL-10 and suppression of IFN $\gamma$ T-cell responses in osteosarcoma patients (49). Furthermore, it has been proposed that expression of TIM-3 on monocytes may be able to shift the balance from IL-12 to IL-23 production and consequently favor type-17 rather than type-1 T-cell responses, driving IL-17mediated inflammation at the expense of anti-pathogen IFN $\gamma$ mediated responses $(50,51)$ (Figure 2). Monocyte expression of TIM-3 is further inducible in response to TLR agonists, including TLR4-mediated LPS stimulation, and this can have a relevant impact in defining the immune milieu in response to bacteria or viruses $(50,51)$. Importantly, blockade of monocyte TIM-3 seems able to reverse the majority of these regulatory or suppressive effects, supporting the restoration of effective immune responses $(48,49,51-53)$

The above-described altered landscape of immunity in advanced liver disease is influenced by checkpoint receptor expression. A paper published in 2015 by one of the authors (AR) was the first to demonstrate that PD-1 and TIM-3 are key in defining this altered immune landscape, and monocyte hyper-stimulation with gut-derived bacterial LPS was found to be the driving factor for these immune dysfunctions (54). We 
observed that adaptive antibacterial T-cell responses in patients with advanced alcohol-related liver disease were prominently skewed toward the production of suppressive IL-10 in response to LPS stimulation, and this was directly correlated with loss of IFN $\gamma$ production and hyper-expression of PD-1 and TIM3 on several immune cell subsets, including T, NK, and NKT cells (54), but not-interestingly-innate-like antibacterial T cells (mucosal-associated invariant T cells, or MAIT) (55). Stimulation with LPS dose-dependently induced PD-1, TIM-3 and IL-10 expression, but blockade of TLR4 and CD14 on monocytes completely abolished these effects; furthermore, blocking PD1 and TIM-3 suppressed IL-10 and restored the production of antibacterial IFN $\gamma$, indicating that the immune defects observed in patients with alcohol-related liver disease may be reversible (54). Similar findings have been described in patients with nonalcoholic sepsis and also in mouse models of sepsis-induced endotoxin-driven liver inflammation [reviewed in (9)].

These results indicate that immune checkpoint blockade may be an effective treatment strategy for the restoration of defective antibacterial immunity in patients with end-stage liver disease. Furthermore, the lack of inflammation observed in our study and the good safety profiles of anti-checkpoint monoclonal antibodies currently used in cancer and sepsis suggest that immune checkpoint blockade may be a safe treatment approach

\section{REFERENCES}

1. Dunham I, Kundaje A, Aldred SF, Collins PJ, Davis CA, Doyle F, et al. (2012). An integrated encyclopedia of DNA elements in the human genome. Nature 489:57-74. doi: 10.1038/nature11247

2. Karlmark KR, Weiskirchen R, Zimmermann HW, Gassler N, Ginhoux F, Weber C, et al. Hepatic recruitment of the inflammatory Grl+ monocyte subset upon liver injury promotes hepatic fibrosis. Hepatology (2009) 50:26174. doi: 10.1002/hep.22950

3. Elsegood CL, Chan CW, Degli-Esposti MA, Wikstrom ME, Domenichini A, Lazarus K, et al. Kupffer cell-monocyte communication is essential for initiating murine liver progenitor cell-mediated liver regeneration. Hepatology (2015) 62:1272-84. doi: 10.1002/hep.27977

4. Ramachandran P, Pellicoro A, Vernon MA, Boulter L, Aucott RL, Ali A, et al. Differential Ly-6C expression identifies the recruited macrophage phenotype, which orchestrates the regression of murine liver fibrosis. Proc Natl Acad Sci USA. (2012) 109:E3186-95. doi: 10.1073/pnas.1119964109

5. Ramachandran P, Iredale JP, Fallowfield JA. Resolution of liver fibrosis: basic mechanisms and clinical relevance. Semin Liver Dis. (2015) 35:119-31. doi: 10.1055/s-0035-1550057

6. Moreau R, Jalan R, Gines P, Pavesi M, Angeli P, Cordoba J, et al. Acute-onchronic liver failure is a distinct syndrome that develops in patients with acute decompensation of cirrhosis. Gastroenterology (2013) 144:1426-37:e1-9. doi: 10.1053/j.gastro.2013.02.042

7. Albillos A, Lario M, Alvarez-Mon M. Cirrhosis-associated immune dysfunction: distinctive features and clinical relevance. J Hepatol. (2014) 61:1385-96. doi: 10.1016/j.jhep.2014.08.010

8. Claria J, Arroyo V, Moreau R. The acute-on-chronic liver failure syndrome, or when the innate immune system goes astray. J Immunol. (2016) 197:3755-61. doi: 10.4049/jimmunol.1600818

9. Riva A, Chokshi S. Immune checkpoint receptors: homeostatic regulators of immunity. Hepatol Int (2018) 12:223-36. doi: 10.1007/s12072-018-9867-9

10. Rimola A, Soto R, Bory F, Arroyo V, Piera C, Rodes J. Reticuloendothelial system phagocytic activity in cirrhosis and its relation to bacterial infections and prognosis. Hepatology (1984) 4:53-8.

11. Albillos A, Hera Ad Ade L, Reyes E, Monserrat J, Munoz L, Nieto M, et al. Tumour necrosis factor-alpha expression by activated monocytes and also in end-stage liver disease, where conventional treatment options are currently very limited.

\section{CONCLUSION}

In conclusion, the innate and adaptive immune systems have many tiers of regulation which have been shown to be dysfunctional in cirrhosis. However, prospectively collected data delineating the time course of immune phenotype by stage of disease in cirrhosis remains scarce. Innate cell reprogramming, through metabolic or epigenetic mechanisms or by targeting checkpoint receptors, remains an attractive area for translational development, although parallel development of reliable immune biomarkers in cirrhosis will be required for immunotherapies to reach their full potential.

\section{AUTHOR CONTRIBUTIONS}

All authors listed have made a substantial, direct and intellectual contribution to the work, and approved it for publication.

\section{FUNDING}

This study was funded by the Foundation for Liver Research. altered T-cell homeostasis in ascitic alcoholic cirrhosis: amelioration with norfloxacin. J Hepatol. (2004) 40:624-31. doi: 10.1016/j.jhep.2003.12.010

12. Gomez F, Ruiz P, Schreiber AD. Impaired function of macrophage Fc gamma receptors and bacterial infection in alcoholic cirrhosis. N Engl J Med. (1994) 331:1122-8. doi: 10.1056/NEJM199410273311704

13. Tazi KA, Quioc JJ, Saada V, Bezeaud A, Lebrec D, Moreau R. Upregulation of TNF-alpha production signaling pathways in monocytes from patients with advanced cirrhosis: possible role of Akt and IRAK-M. J Hepatol. (2006) 45:280-9. doi: 10.1016/j.jhep.2006.02.013

14. Wasmuth HE, Kunz D, Yagmur E, Timmer-Stranghoner A, Vidacek $\mathrm{D}$, Siewert E, et al. Patients with acute on chronic liver failure display "sepsis-like" immune paralysis. J Hepatol. (2005) 42:195-201. doi: 10.1016/j.jhep.2004.10.019

15. Gandoura S, Weiss E, Rautou PE, Fasseu M, Gustot T, Lemoine F, et al. Geneand exon-expression profiling reveals an extensive LPS-induced response in immune cells in patients with cirrhosis. $J$ Hepatol. (2013) 58:936-48. doi: 10.1016/j.jhep.2012.12.025

16. O'Brien AJ, Fullerton JN, Massey KA, Auld G, Sewell G, James S, et al. Immunosuppression in acutely decompensated cirrhosis is mediated by prostaglandin E2. Nat Med. (2014) 20:518-23. doi: 10.1038/nm.3516

17. Bernsmeier C, Pop OT, Singanayagam A, Triantafyllou E, Patel VC, Weston $\mathrm{CJ}$, et al. Patients with acute-on-chronic liver failure have increased numbers of regulatory immune cells expressing the receptor tyrosine kinase MERTK. Gastroenterology (2015) 148:603-15.e14. doi: 10.1053/j.gastro.2014.11.045

18. Ginhoux F, Jung S. Monocytes and macrophages: developmental pathways and tissue homeostasis. Nat Rev Immunol. (2014) 14:392-404. doi: 10.1038/nri3671

19. Ziegler-Heitbrock L. The CD14+ CD16+ blood monocytes: their role in infection and inflammation. J Leukoc Biol. (2007) 81:584-92. doi: $10.1189 / \mathrm{jlb} .0806510$

20. Sunderkotter C, Nikolic T, Dillon MJ, Van Rooijen N, Stehling M, Drevets DA, et al. Subpopulations of mouse blood monocytes differ in maturation stage and inflammatory response. J Immunol. (2004) 172:4410-7. doi: 10.4049/jimmunol.172.7.4410

21. Serbina NV, Pamer EG. Monocyte emigration from bone marrow during bacterial infection requires signals mediated by chemokine receptor CCR2. Nat Immunol. (2006) 7:311-7. doi: 10.1038/ni1309 
22. Tsou CL, Peters W, Si Y, Slaymaker S, Aslanian AM, Weisberg SP, et al. Critical roles for CCR2 and MCP-3 in monocyte mobilization from bone marrow and recruitment to inflammatory sites. J Clin Invest. (2007) 117:9029. doi: 10.1172/JCI29919

23. Swirski FK, Nahrendorf M, Etzrodt M, Wildgruber M, Cortez-Retamozo $\mathrm{V}$, Panizzi $\mathrm{P}$, et al. Identification of splenic reservoir monocytes and their deployment to inflammatory sites. Science (2009) 325:612-6. doi: 10.1126/science.1175202

24. Patel AA, Zhang Y, Fullerton JN, Boelen L, Rongvaux A, Maini AA, et al. The fate and lifespan of human monocyte subsets in steady state and systemic inflammation. J Exp Med. (2017) 214:1913-23. doi: 10.1084/jem.20170355

25. Zigmond E, Samia-Grinberg S, Pasmanik-Chor M, Brazowski E, Shibolet O, Halpern $\mathrm{Z}$, et al. Infiltrating monocyte-derived macrophages and resident kupffer cells display different ontogeny and functions in acute liver injury. $J$ Immunol. (2014) 193:344-53. doi: 10.4049/jimmunol.1400574

26. Dal-Secco D, Wang J, Zeng Z, Kolaczkowska E, Wong CH, Petri B, et al. A dynamic spectrum of monocytes arising from the in situ reprogramming of CCR2 + monocytes at a site of sterile injury. J Exp Med. (2015) 212:447-56. doi: 10.1084/jem.20141539

27. Bernsmeier C, Triantafyllou E, Brenig R, Lebosse FJ, Singanayagam A, Patel VC, et al. CD14(+) CD15(-) HLA-DR(-) myeloid-derived suppressor cells impair antimicrobial responses in patients with acute-on-chronic liver failure. Gut (2018) 67:1155-67. doi: 10.1136/gutjnl-2017-314184

28. Wang J, Kubes P. A reservoir of mature cavity macrophages that can rapidly invade visceral organs to affect tissue repair. Cell (2016) 165:668-78. doi: 10.1016/j.cell.2016.03.009

29. Netea MG, Quintin J, van der Meer JW. Trained immunity: a memory for innate host defense. Cell Host Microbe (2011) 9:355-61. doi: 10.1016/j.chom.2011.04.006

30. Saeed S, Quintin J, Kerstens HH, Rao NA, Aghajanirefah A, Matarese $\mathrm{F}$, et al. Epigenetic programming of monocyte-to-macrophage differentiation and trained innate immunity. Science (2014) 345:1251086. doi: $10.1126 /$ science. 1251086

31. Weis S, Carlos AR, Moita MR, Singh S, Blankenhaus B, Cardoso S, et al. Metabolic adaptation establishes disease tolerance to sepsis. Cell (2017) 169:1263-75.e14. doi: 10.1016/j.cell.2017.05.031

32. Medzhitov R, Schneider DS, Soares MP. Disease tolerance as a defense strategy. Science (2012) 335:936-41. doi: 10.1126/science.1214935

33. Carre JE, Orban JC, Re L, Felsmann K, Iffert W, Bauer M, et al. Survival in critical illness is associated with early activation of mitochondrial biogenesis. Am J Respir Crit Care Med. (2010) 182:745-51. doi: 10.1164/rccm.201003-0326OC

34. Foster SL, Hargreaves DC, Medzhitov R. Gene-specific control of inflammation by TLR-induced chromatin modifications. Nature (2007) 447:972-8. doi: 10.1038/nature05836

35. Chan C, Li L, McCall CE, Yoza BK. Endotoxin tolerance disrupts chromatin remodeling and NF-kappaB transactivation at the IL-1beta promoter. $J$ Immunol. (2005) 175:461-8. doi: 10.4049/jimmunol.175.1.461

36. El Gazzar M, Yoza BK, Chen X, Garcia BA, Young NL, McCall CE. Chromatin-specific remodeling by HMGB1 and linker histone $\mathrm{H} 1$ silences proinflammatory genes during endotoxin tolerance. Mol Cell Biol. (2009) 29:1959-71, doi: 10.1128/MCB.01862-08

37. De Santa F, Totaro MG, Prosperini E, Notarbartolo S, Testa G, Natoli G. The histone H3 lysine-27 demethylase Jmjd3 links inflammation to inhibition of polycomb-mediated gene silencing. Cell (2007) 130:1083-94. doi: 10.1016/j.cell.2007.08.019

38. Liu TF, Yoza BK, El Gazzar M, Vachharajani VT, McCall CE. NAD+dependent SIRT1 deacetylase participates in epigenetic reprogramming during endotoxin tolerance. J Biol Chem. (2011) 286:9856-64. doi: 10.1074/jbc.M110.196790

39. Tao J, Zhang J, Ling Y, McCall CE, Liu TF. Mitochondrial sirtuin 4 resolves immune tolerance in monocytes by rebalancing glycolysis and glucose oxidation homeostasis. Front Immunol. (2018) 9:419. doi: 10.3389/fimmu.2018.00419

40. Bekkering S, Blok BA, Joosten LA, Riksen NP, van Crevel R, Netea MG. In Vitro experimental model of trained innate immunity in human primary monocytes. Clin Vaccine Immunol. (2016) 23:926-33. doi: 10.1128/CVI.00349-16
41. Vachharajani VT, Liu T, Brown CM, Wang X, Buechler NL, Wells JD, et al. SIRT1 inhibition during the hypoinflammatory phenotype of sepsis enhances immunity and improves outcome. J Leukoc Biol. (2014) 96:785-96. doi: 10.1189/jlb.3MA0114-034RR

42. Wang X, Buechler NL, Martin A, Wells J, Yoza B, McCall CE, et al. Sirtuin-2 Regulates Sepsis Inflammation in ob/ob Mice. PLoS ONE (2016) 11:e0160431. doi: 10.1371/journal.pone.0160431

43. Carpenter S, Aiello D, Atianand MK, Ricci EP, Gandhi P, Hall LL, et al. A long noncoding RNA mediates both activation and repression of immune response genes. Science (2013) 341:789-92. doi: 10.1126/science.1240925

44. Said EA, Dupuy FP, Trautmann L, Zhang Y, Shi Y, El-Far M, et al. Programmed death-1-induced interleukin-10 production by monocytes impairs CD4+ T cell activation during HIV infection. Nat Med. (2010) 16:452-9. doi: 10.1038/nm.2106

45. Tai H, Xing H, Xiang D, Zhu Z, Mei H, Sun W, et al. Monocyte programmed death Ligand-1, A Predicator for 28-day mortality in septic patients. Am J Med Sci. (2018) 355:362-7. doi: 10.1016/j.amjms.2017.12.008

46. Zasada M, Lenart M, Rutkowska-Zapala M, Stec M, Durlak W, Grudzien A, et al. Analysis of PD-1 expression in the monocyte subsets from non-septic and septic preterm neonates. PLoS ONE (2017) 12:e0186819. doi: 10.1371/journal.pone.0186819

47. Wang Z, Yin N, Zhang Z, Zhang Y, Zhang G, Chen W. Upregulation of T-cell Immunoglobulin and mucin-domain containing-3 (Tim-3) in monocytes/macrophages associates with gastric cancer progression. Immunol Invest. (2017) 46:134-48. doi: 10.1080/08820139.2016. 1229790

48. Hou N, Jiang N, Zou Y, Piao X, Liu S, Li S, et al. Down-regulation of tim-3 in monocytes and macrophages in plasmodium infection and its association with parasite clearance. Front Microbiol. (2017) 8:1431. doi: 10.3389/fmicb.2017.01431

49. Li X, Chen Y, Liu X, Zhang J, He X, Teng G, et al. Tim3/Gal9 interactions between $T$ cells and monocytes result in an immunosuppressive feedback loop that inhibits Th1 responses in osteosarcoma patients. Int Immunopharmacol. (2017) 44:153-9. doi: 10.1016/j.intimp.2017.01.006

50. Ma CJ, Li GY, Cheng YQ, Wang JM, Ying RS, Shi L, et al. Cis association of galectin-9 with Tim-3 differentially regulates IL-12/IL-23 expressions in monocytes via TLR signaling. PLoS ONE (2013) 8:e72488. doi: 10.1371/journal.pone.0072488

51. Zhang Y, Ma CJ, Wang JM, Ji XJ, Wu XY, Jia ZS, et al. Tim-3 negatively regulates IL-12 expression by monocytes in HCV infection. PLoS ONE (2011) 6:e19664. doi: 10.1371/journal.pone.0019664

52. Wang JM, Ma CJ, Li GY, Wu XY, Thayer P, Greer P, et al. Tim-3 alters the balance of IL-12/IL-23 and drives TH17 cells: role in hepatitis B vaccine failure during hepatitis C infection. Vaccine (2013) 31:2238-45. doi: 10.1016/j.vaccine.2013.03.003

53. Wang JM, Shi L, Ma CJ, Ji XJ, Ying RS, Wu XY, et al. Differential regulation of interleukin-12 (IL-12)/IL-23 by Tim-3 drives $\mathrm{T}(\mathrm{H}) 17$ cell development during hepatitis C virus infection. J Virol. (2013) 87:4372-83. doi: 10.1128/jvi.03376-12

54. Markwick LJL, Riva A, Ryan JM, Cooksley H, Palma E, Tranah TH, et al. Blockade of PD1 and TIM3 restores innate and adaptive immunity in patients with acute alcoholic hepatitis. Gastroenterology (2015) 148:590602.e10. doi: 10.1053/j.gastro.2014.11.041

55. Riva A, Patel V, Kurioka A, Jeffery HC, Wright G, Tarff S, et al. Mucosaassociated invariant $\mathrm{T}$ cells link intestinal immunity with antibacterial immune defects in alcoholic liver disease. Gut (2018) 67:918-30. doi: 10.1136/gutjnl-2017-314458

Conflict of Interest Statement: The authors declare that the research was conducted in the absence of any commercial or financial relationships that could be construed as a potential conflict of interest.

Copyright (c) 2019 Riva and Mehta. This is an open-access article distributed under the terms of the Creative Commons Attribution License (CC BY). The use, distribution or reproduction in other forums is permitted, provided the original author(s) and the copyright owner(s) are credited and that the original publication in this journal is cited, in accordance with accepted academic practice. No use, distribution or reproduction is permitted which does not comply with these terms. 